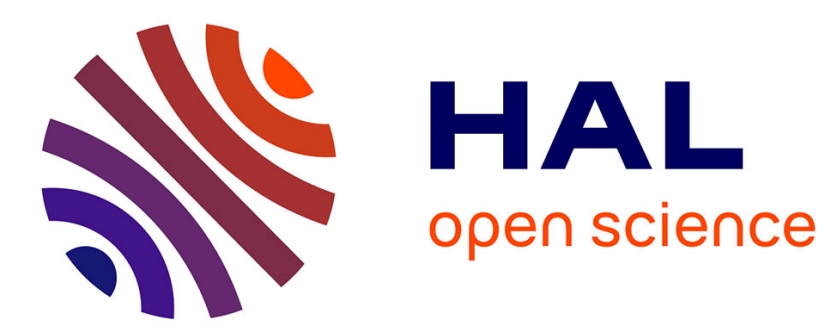

\title{
Tau-sterile neutrino mixing in NOMAD
}

P. Nedelec

\section{- To cite this version:}

P. Nedelec. Tau-sterile neutrino mixing in NOMAD. International workshop on tau lepton physics 6, Sep 2000, Victoria, Canada. pp.37-42. in2p3-00010929

\section{HAL Id: in2p3-00010929 https://hal.in2p3.fr/in2p3-00010929}

Submitted on 12 Mar 2002

HAL is a multi-disciplinary open access archive for the deposit and dissemination of scientific research documents, whether they are published or not. The documents may come from teaching and research institutions in France or abroad, or from public or private research centers.
L'archive ouverte pluridisciplinaire HAL, est destinée au dépôt et à la diffusion de documents scientifiques de niveau recherche, publiés ou non, émanant des établissements d'enseignement et de recherche français ou étrangers, des laboratoires publics ou privés. 
LAPP-EXP 2000-13

December 2000

\title{
Tau-sterile neutrino mixing in NOMAD
}

\author{
P. NEDELEC \\ LAPP, IN2P3-CNRS, Chemin de Bellevue, BP110, \\ F-74941, Annecy-le-Vieux
}

\begin{abstract}
For several years, the KARMEN experiment was reporting an anomalous signal which was interpreted as a possible existence of heavy sterile neutrinos. The NOMAD experiment has the capability to search for such an isosinglet neutrino. It is assumed that the $\left(\nu_{s}\right)$ couples to the $\nu_{\tau}$ produced in the SPS neutrino beam at CERN. Analysis of NOMAD data leads to a single candidate event, compatible with the background expectations. This allows a limit to be set for the first time on the mixing strength between sterile and tau neutrinos, in the mass range 10 to $190 \mathrm{MeV}$.
\end{abstract}

Talk given at the TAU2000 Workshop, 18-21 September 2000, Victoria, Canada 


\title{
Tau-sterile neutrino mixing in NOMAD
}

\author{
P. Nédélec ${ }^{\mathrm{a}}$

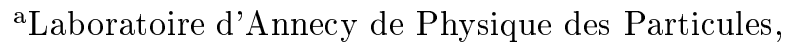 \\ chemin de Bellevue, 74941 Annecy-le-Vieux CEDEX, France
}

For several years, the KARMEN experiment was reporting an anomalous signal which was interpreted as a possible existence of heavy sterile neutrinos. The NOMAD experiment has the capability to search for such an isosinglet neutrino. It is assumed that the $\left(\nu_{s}\right)$ couples to the $\nu_{\tau}$ produced in the SPS neutrino beam at CERN. Analysis of NOMAD data leads to a single candidate event, compatible with the background expectations. This allows a limit to be set for the first time on the mixing strength between sterile and tau neutrinos, in the mass range 10 to $190 \mathrm{MeV}$.

\section{Introduction}

In the Standard Model (SM) of Particle Physics, neutrinos only interact via their $\mathrm{SU}(2)_{L}$ left-handed states. In several models beyond the SM, like Grand Unified Theories and Superstrings inspired models [1], heavy isosinglet neutrinos $\left(\nu_{s}\right)$ not coupling to the $\mathrm{W}$ and $\mathrm{Z}$ bosons are naturally introduced. Such sterile neutrinos are also introduced in other models developed to solve baryo/leptogenesis in the Universe, like left-right symmetric or see-saw models. Their masses are predicted in the $\mathrm{GeV}$ - TeV range. Light sterile neutrinos, $m_{\nu}<e V$, are introduced [2] to explain in a coherent framework the solar, the atmospheric (SuperK) and LSND neutrino experiment's results. The different experiments explain their observations via a neutrino oscillations mechanism. This implies three different $\Delta m^{2}$. Since LEP has limited the number of ligth neutrinos to 3 , a fourth sterile neutrino $\nu_{s}$ is an elegant way to solve the neutrino puzzle.

Recently the KARMEN experiment was reporting about an anomaly in the time structure of its events, which was originally interpreted as an indication of a heavy neutral particle in the few tens $\mathrm{MeV}$ range [3]. More recently a KARMEN representative reported on "no more indication of an oscillation" [4].

The NOMAD experiment at CERN, has the capability to look for such heavy neutral particle for masses up to few a $100 \mathrm{MeV}$ [5]

The main question concerning a new heavy neutrino is to know if it couples to ordinary neutrinos and if so, what are the mixing parameters $U_{l i}$. Leptonic decay and neutrino experiments set limits on $\left|U_{e s}\right|^{2}$ and $\left|U_{\mu s}\right|^{2}$. Concerning $\left|U_{\tau s}\right|^{2}$, no experimental limits exist in the $100 \mathrm{MeV}$ mass region. Recently, cosmology and astrophysical considerations were used to constrain $\left|U_{\tau s}\right|^{2}$ in this mass region [9].

\section{The KARMEN anomaly}

The KARMEN experiment studies the interactions of neutrinos in a large tank filled with 56 tons of liquid scintillator. Neutrinos are produced by stopping $800 \mathrm{MeV}$ protons in a massive target, producing pions. The $\pi^{-}$are absorbed by the target while the $\pi^{+}$decay at rest (DAR) via $\pi^{+} \rightarrow \mu^{+} \nu_{\mu}$. The low momentum $\mu^{+}$also stops in the target and then decays via $\mu^{+} \rightarrow e^{+} \overline{\nu_{\mu}} \nu_{e}$.

The specific time structure of the beam (two pulses, $100 \mathrm{~ns}$ long $225 \mathrm{~ns}$ apart) allows for discrimination between neutrinos coming from $\pi$ decaying at rest ( $\tau=26 \mathrm{~ns}$ ) and the neutrinos coming from the subsequent $\mu$ decay $(\tau=2.2 \mu \mathrm{s})$, as indicated on Figure 1. Due to the $\pi-\mu$ chain, the neutrino beam contains identical intensities of $\nu_{\mu}, \bar{\nu}_{\mu}$ and $\nu_{e}$. The fiducial region of the detector is situated between $16.6 \mathrm{~m}$ and $19.8 \mathrm{~m}$ from the neutrino source. The detector is surrounded by a 


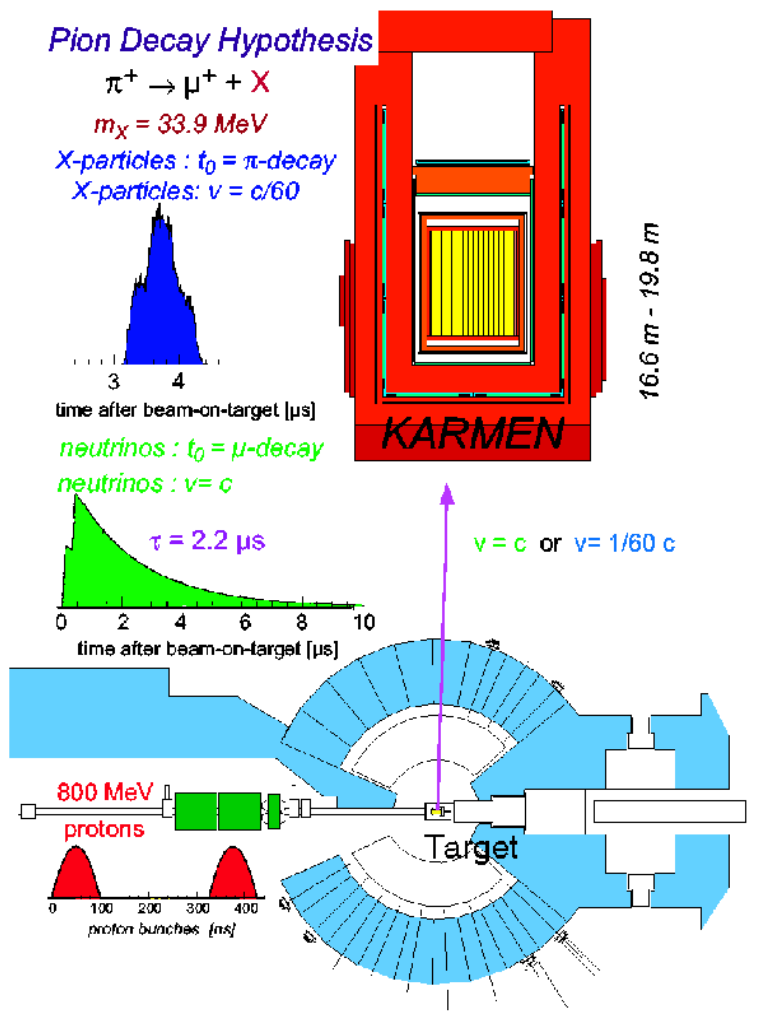

Figure 1. The KARMEN experimental setup

huge shielding system, made out of 7000 tons of steel equipped with two layers of veto counters, in order to suppress backgrounds from beam correlated spallation neutrons, from hadrons from cosmic radiation and to reduce cosmic muon flux. This configuration is know as KARMEN1. In 1996 a third, large area, veto system $\left(300 \mathrm{~m}^{2}\right)$ was installed to further improve the shielding (KARMEN2 experimental configuration).

In the time window $0.6-10.6 \mu \mathrm{s}$, after the beam is incident on the target, one expects a signal distribution corresponding to the $2.2 \mu$ s lifetime of the muon superimposed on a flat cosmic background. The KARMEN1 data exhibit a bumplike distortion in the time spectrum around 3.1 $4.1 \mu \mathrm{s}$, the so-called anomaly, as shown on Figure 2. This excess was intepreted as coming from the decay of slow-moving, neutral, weakly interacting

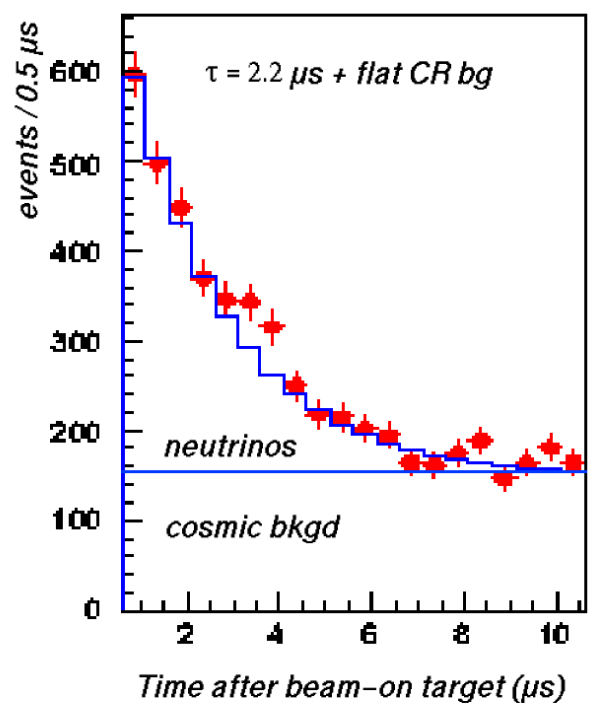

Figure 2. Time distribution of KARMEN events with the anomaly bump

particle $\mathrm{X}$ inside the fiducial volume, where $\mathrm{X}$ is produced in a rare decay of pions: $\pi^{+} \rightarrow \mu^{+} X$. The mass of this new particle was estimated to be $m_{X} \sim 33.9 \mathrm{MeV}$. An analysis including a first part of the KARMEN2 data sample confirmed the accumulation of events. However, an updated analysis of all the KARMEN2 data, collected so far (March 2000) revealed no more distortion in the time distribution. Based on the incompatibility between the two data periods, the KARMEN experiment concluded that the $\pi^{+} \rightarrow \mu^{+} X$ hypothesis had to be rejected.

In NOMAD we have initiated an analysis to look for such an effect. Let me first describe the principle of the search.

\section{Principle of the search}

As schematically shown on Figure 3, the sterile neutrino $\nu_{s}$ is produced in the $D_{s} \rightarrow \tau \nu_{s}$ decay through $\nu_{s}-\nu_{\tau}$ mixing. The $D_{s}$ meson is produced in the $p+B e$ interactions at the neutrino target. The $\nu_{s}$ propagates to NOMAD through the downstream neutrino beam shielding without significant attenuation if it is a long-lived particle. Occasionally a $\nu_{s}$ decays in the NOMAD detector via the process : $\nu_{s} \rightarrow \nu_{\tau} e^{+} e^{-}$. An excess 


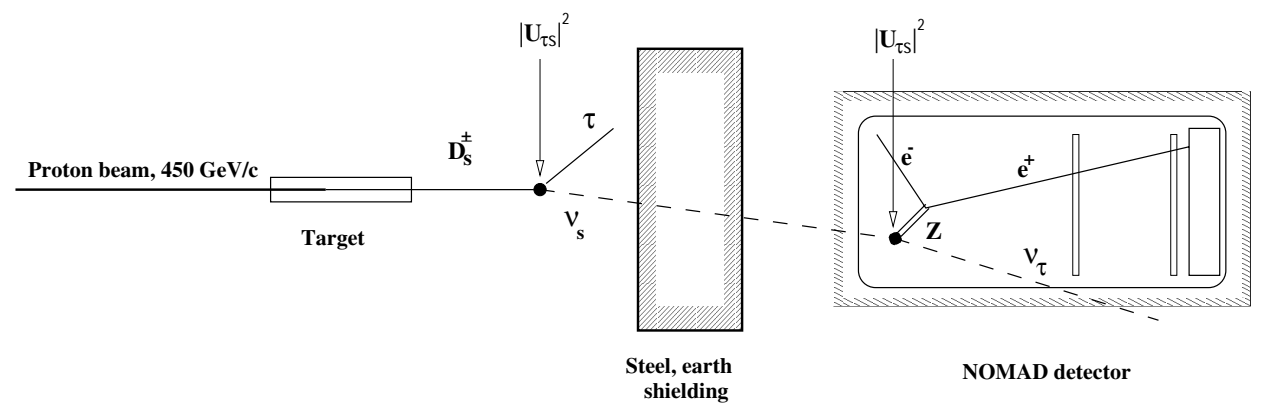

Figure 3. Principle of a sterile neutrino $\nu_{s}$ production, propagation in the beam dump and interaction in the NOMAD detector.

of $e^{+} e^{-}$pairs events above the expected background would indicate the presence of a $\nu_{\tau}-\nu_{s}$ in the neutrino beam.

\section{The NOMAD experiment}

The NOMAD detector is described elsewhere [6]. It consists of different sub-detectors installed in a $0.4 \mathrm{~T}$ dipole magnet: a set of drift chambers (DC) as active target (2.7 tons), a high performance transition radiation detector (TRD) [7] for particle identification and a calorimetric system for energy measurements. Outside the magnetic volume, a hadronic calorimeter and a set of muon chambers complete the experiment. The electron identification is provided mainly by the TRD which has a rejection power for isolated electrons greater than $10^{3}$ at $90 \%$ efficency for the electron in the momentum range $1-5 \mathrm{GeV}[8]$.

\section{Event selection}

The data used for this $\nu_{s} \rightarrow \nu_{\tau} e^{+} e^{-}$analysis correspond to the full $\bar{V} T 1 T 2$ data sample collected during the period 1996-1998. This data set corresponds to a total of $4.1 \times 10^{19}$ protons on target (pot's). The signal events are characterised by a $e^{+} e^{-}$pair with no other activity in the detector. The selection criteria for the selection of the signal are the following:

- Event in NOMAD fiducial volume: two tracks at the primary vertex, at least one of them identified as an electron by the TRD; $E_{\text {pair }}>4 \mathrm{GeV}$; invariant mass $m_{\text {pair }}<95$ $\mathrm{MeV}$.

- Calorimetric activity: no photons with $E_{\gamma}>0.4 \mathrm{GeV}$, except if compatible with a $e^{+}$or $e^{-}$bremsstrahlung photon.

- No other activity: $E_{H C A L}<0.4 \mathrm{GeV}$; no muon in the event.

After this selection 207 events are collected. Because of the high collinearity of the $e^{+} e^{-}$pair with the neutrino beam for the signal, an angular cut is applied to further reduce the background. One defines the variable $\mathcal{C} \equiv 1-\cos \Theta_{\nu\left(e^{+} e^{-}\right)}$, where $\Theta_{\nu\left(e^{+} e^{-}\right)}$is the angle between the neutrino beam direction and the total momentum of the reconstructed $e^{+} e^{-}$pair. The cut applied to select signal in the reference box is $\mathcal{C}<2 \times 10^{-5}$.

The accuracy of the colinearity determination for simulation events was checked with a sample of $\nu_{\mu} \mathrm{CC}$ data having a $e^{+} e^{-}$pair coming from a photon conversion, produced far from the main vertex.

\section{Backgrounds}

The main background comes from neutrino interactions in the surrounding material of the NOMAD target: the return yokes, the front pillar and the magnetic coil, producing a single $\pi^{0}$ in the fiducial volume, the other particles remaining 
outside the sensitive region. So the background events are characterised by a $e^{+} e^{-}$pair in the target region coming from the $\pi^{0}$ decay, with very little hadronic activity. This background has been estimated, based on a full simulation of neutrino interactions in the overall detector.

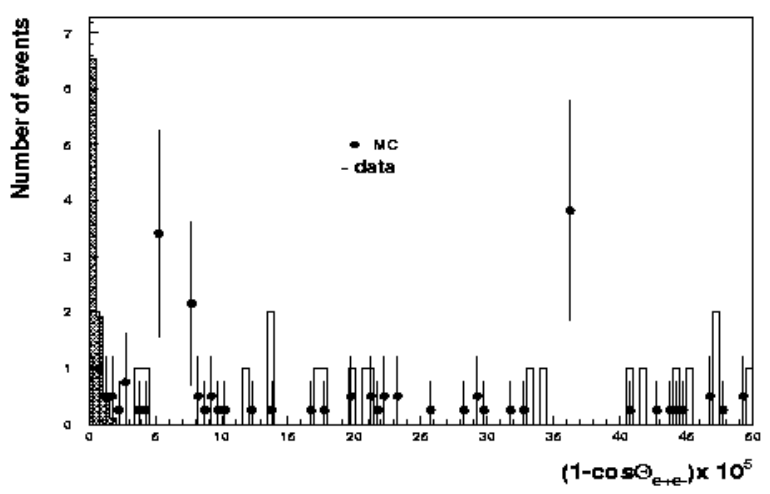

Figure 4. Distribution of $\mathcal{C} \equiv 1-\cos \Theta_{\nu\left(e^{+} e^{-}\right)}$ for data and Monte-Carlo. Shaded area (narrow peak on the left side) represents the region of the expected signal

\section{Results and limits}

In order to avoid human bias in the selection criteria a blind analysis has been performed. This means that data and Monte-Carlo events were compared in a region where no signal in expected, i.e. outside the box as shown on Figure 4. Outside the box, 19 events are observed and $20 \pm 4$ are predicted.

Two different methods are used to estimate the background in the signal box, the first one is based on Monte-Carlo, while the other relies only on the data themselves. The two methods give consistent results: $N_{b k g}^{\mathrm{MC}}=2.5_{-0.8}^{+0.9}($ stat $) \pm$ 0.6 (syst) events from the $\mathrm{MC}$ and $N_{b k g}^{\text {Data }}=$ $2.4_{-0.9}^{+0.9}($ stat $) \pm 0.7$ (syst) events from the data, where the systematic error includes uncertainties on the luminosity ( $5 \%$ on the number of pot's) and on the coherent $\pi^{0}$ production cross section $(25 \%)$. Upon opening the box, we have found one event passing the selection cuts. This is consistent with the expected background and hence no evidence for isosinglet neutrino decays has been found.

We can then determine the 90\% C.L. upper limit for the corresponding mixing amplitude $\left|U_{\tau s}\right|^{2}$ as a function of the $\nu_{s}$ mass by using the following relation:

$N_{\nu_{s} \rightarrow \nu_{\tau} e^{+} e^{-}}<N_{\nu_{s} \rightarrow \nu_{\tau} e^{+} e^{-}}^{\mathrm{up}}$

The $90 \%$ C.L. upper limit for the expected number of signal events, $N_{\nu_{s} \rightarrow \nu_{\tau} e^{+} e^{-}}^{\text {up }}$, is computed within the frequentist approach of ref [10] by taking into account the uncertainties in the background estimate [11]. This results in $N_{\nu_{s} \rightarrow \nu_{\tau} e^{+} e^{-}}^{\text {up }}=2.1$ events.

For a given flux $\Phi\left(\nu_{s}\right)$, the expected number of $\nu_{s} \rightarrow \nu_{\tau} e^{+} e^{-}$decays occuring within the fiducial length $L$ of the NOMAD detector located at a distance $L^{\prime}$ from the neutrino target is given by

$$
\begin{array}{r}
N_{\nu_{s} \rightarrow \nu_{\tau} e^{+} e^{-}}=\int \Phi\left(\nu_{s}\right) \cdot \exp \left(-L^{\prime} m_{\nu_{s}} / p_{\nu_{s}} \tau_{s}\right) \cdot \\
{\left[1-\exp \left(-L m_{\nu_{s}} / p_{\nu_{s}} \tau_{s}\right)\right]} \\
\cdot\left(\Gamma_{e^{+} e^{-}} / \Gamma_{t o t}\right) \cdot \varepsilon \cdot A \cdot d E_{\nu_{s}} \propto\left|U_{\tau s}\right|^{4}
\end{array}
$$

where $p_{\nu_{s}}$ is the $\nu_{s}$ momentum, $\Gamma_{e^{+} e^{-}}, \Gamma_{t o t}$ are the partial and total mass dependent $\nu_{s}$-decay widths, and $\varepsilon$ is the $e^{+} e^{-}$pair reconstruction efficiency. The acceptance $A$ of the NOMAD detector was calculated tracing $\nu_{s}$ 's produced in the Be-target through the beam dump to the detector taking all relevant momentum and angular distributions into account [12]. The flux $\Phi\left(\nu_{s}\right)$ of sterile neutrinos can be related to the flux of $\nu_{\tau}$ present in the SPS neutrino beam [5].

The final $90 \%$ C.L. upper limit curve in the $\left(m_{\nu_{s}} ;\left|U_{\tau s}\right|^{2}\right)$ plane is shown in Figure 5 together with the Big Bang Nucleosynthesis (BBN) and supernovae SN1987a lower limits obtained in ref. [9]. For the mass range $m_{\nu_{s}} \geq m_{\pi}$ the shape of the NOMAD limit curve is explained by the contribution from the $\nu_{s} \rightarrow \nu_{\tau} \pi^{0}$ decay to the $\nu_{s}$ decay rate: as the $\nu_{s} \rightarrow \nu_{\tau} \pi^{0}$ decay channel opens 


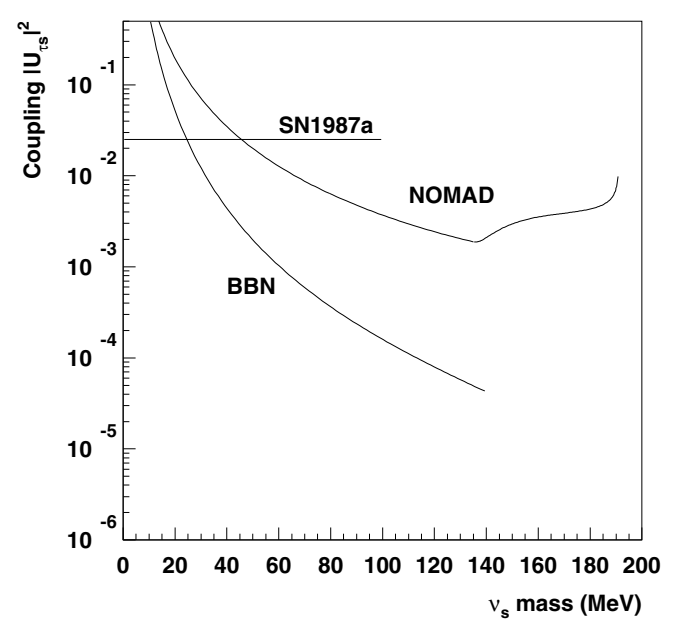

Figure 5. Exclusion regions for $\left|U_{\tau s}\right| 2$ vs $\mathrm{m}_{s}$

up, the branching ratio $\mathrm{BR}\left(\nu_{s} \rightarrow \nu_{\tau} e^{+} e^{-}\right)$drops rapidly as $m_{\nu_{s}}$ increases.

Our data can also be used to restrict one of the interpretations of a time anomaly observed by the KARMEN experiment. From our limit curve in Figure 5 we can give for each mass value a limit on the coupling strength. This limit can be converted to a lifetime constraint. For $m_{\nu_{s}}=33.9 \mathrm{MeV}$ the lifetime of such a neutrino has to be greater than $\simeq 10^{-2}$ seconds, as indicated on Figure 6 . The BBN lower limit of ref. [13] constrains the $\nu_{s}$ lifetime to be less than 0.1 sec. This results in a small window around $B R\left(\pi^{+} \rightarrow \mu^{+}+X\right) \simeq 10^{-12}$ left untested. Note, that the recent PSI result on the search for the $33.9 \mathrm{MeV}$ particle in $\pi^{+} \rightarrow \mu^{+}+X$ decay corresponds to $B R\left(\pi^{+} \rightarrow \mu^{+}+X\right)<6.0 \cdot 10^{-10}$ at $95 \%$ C.L. [15].

\section{Conclusion}

In NOMAD we have carried out a search for isosinglet heavy neutrinos $\left(\nu_{s}\right)$ produced in the $D_{s} \rightarrow \tau \nu_{\tau}$ decay through $\nu_{\tau}-\nu_{s}$ mixing, followed by a decay $\nu_{s} \rightarrow \nu_{\tau} e^{+} e^{-}$in the detector.

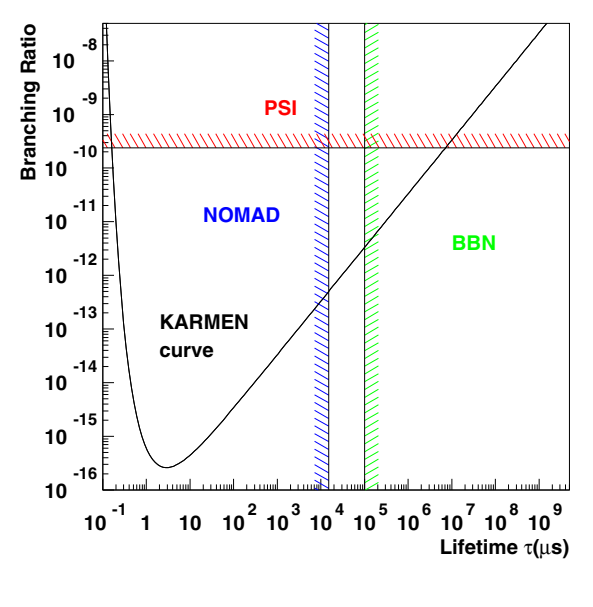

Figure 6. Exclusion regions for $B r$ vs lifetime

The excellent capabilities of the apparatus for the identification and the measurement of the $e^{+} e^{-}$ pair direction allow substancial suppression of the background from standard processes. A single candidate event, consistent with expectations was found. This allows to derivation of limits on mixing strength $\left|U_{\tau s}\right|^{2}$ in the masse range 10-190 $\mathrm{MeV}$ to be made. The results obtained are used to constrain the interpretation of the KARMEN anomaly in terms of a rare decay $\pi^{+} \rightarrow \mu^{+}+X$, where $X$ has a mass of $33.9 \mathrm{MeV}$ and is either a isosinglet neutrino $\nu_{s}$ or a new, short-lived weakly interacting neutral fermion. A $90 \%$ C.L. lower limit on the $\nu_{s}$ lifetime $\tau_{\nu_{s}}>0.017 \mathrm{sec}$ is obtained. The window $0.017<\tau_{\nu_{s}}<0.1 s$ between the BBN upper limit and present lower limit is still open for future experimental searches.

\section{9. acknowledgements}

I would like to thank my NOMAD colleagues with whom we made this nice experiment and particularly L. DiLella and S. Gninenko for valuable discussions. 


\section{REFERENCES}

1. R.N. Mohapatra and P.B. Pal, in "Massive Neutrinos in Physics and Astrophysics", World Scientific, Singapore, 1991.

2. O.V. Mazurin and E.A. Porai-Koshits (eds.), Phase Separation in Glass, North-Holland, Amsterdam, 1984.

3. J-M Levy and F. Vannucci, A consistent interpretation of the KARMEN anomaly, hep$\mathrm{ph} / 0003194$ (2000)

S.N. Gninenko and N..K Krasnikov, Exotic muon decays and the KARMEN anomaly, hep-ph/9804364v4 (1998).

4. K. Eitel, Latest results of the KARMEN2 Experiment, hep-ph/0008002 (2000).

5. P. Astier et al (NOMAD Collab.), Search for $\mathrm{MeV}$ isosinglet neutrinos mixing to tau neutrinos, CERN-EP-xxx (2000)

6. J. Altegoer et al (NOMAD Collab.), The NOMAD experiment at the CERN SPS, Nucl. Instr. and Meth. A404 (1998) 96.

7. G. Bassompierre et al, A large area transition transition detector for the NOMAD experiment, Nucl. Instr. and Meth. A403 (1998) 363.

8. G. Bassompierre et al, Performance of the NOMAD transition radiatio detector, Nucl. Instr. and Meth. A411 (1998) 63.

9. A.D. Dolgov, S.H. Hansen, G. Raffelt, and D.V. Semikoz, Preprint hep-ph/0008138, Submitted to Nucl. Phys. B.

10. G.J. Feldman and R.D. Cousins, Phys. Rev. D 57 (1998) 3873.

11. A. Stuart and J.K. Ord, "Kendall's Advanced Theory of Statistics", vol. 2, "Classical Inference and Relationship, 5th ed., Oxford Univ. Press, New York, 1991, p.861, Eq.(23.4).

12. M.C. Gonzalez-Garcia and J.J. GomezCadenas, Phys. Rev. D 55 (1997) 1297.

13. A. D. Dolgov, S. H. Hansen, G. Raffelt, and D. V. Semikoz, Nucl. Phys. B 580 (2000) 331.

14. V. Barger, R.J.N. Phillips and S. Sarkar, Phys. Lett. B352 (1995) 365;

(E) ibid. B 356 (1995) 671.

15. M. Daum et al., Phys. Rev. Lett. 85 (2000)
1815.

16. F. Atchison, M. Daum, P.R. Kettle, C. Wigger, Preprint hep-ex/0008073. 